\section{Effects of ACE inhibition on sodium excretion in COPD}

The limitations of angiotensin converting enzyme (ACE) inhibitors in promoting natriuresis in patients with cor pulmonale discussed by Dr A G Stewart and colleagues (October 1994;49:995-8) should not detract from the potential benefits of ACE blockade in retarding deterioration of left ventricular systolic function in those patients who, as a result of mediation of cigarette smoking in the pathogenesis of chronic obstructive pulmonary disease (COPD) and coronary heart disease, respectively, happen to have left ventricular systolic failure as a coexisting disorder. We should be more vigilant in documenting the association of COPD and left ventricular systolic dysfunction ${ }^{1}$ in patients with cor pulmonale because co-prescription of long term oxygen therapy for cor pulmonale and ACE inhibitors for left ventricular systolic failure could improve life expectancy, especially when associated with cessation of cigarette smoking.

O M P JOLOBE Department of Medicine for the Elderly Tameside General Hospital, Ashton under Lyne Lancashire OL6 9RW,

$U K$

1 Steele P, van Dyke D, Sutton F, et al. Left ventricular ejection fraction in severe chronic obstructive airways disease. Am $\mathcal{F}$ Med 1975 59:21-8.

2 The SOLVD Investigators. Effect of enalapril on mortality and development of heart failure in asymptomatic patients with reduced left in asympth reduced left 1992;327:685-91.

AUTHOR'S REPLY The primary aim of our paper was to investigate the potential role of aldosterone as the cause of the sodium retention seen in patients with hypoxaemic COPD. The ACE inhibitor perindopril was used as a tool to lower the raised aldosterone levels. Although chronic ACE inhibition did not improve sodium excretion, this does not detract from its potential use in the acute situation. ${ }^{1}$ We did not investigate the potential effects of ACE inhibition on well being, dyspnoea, exercise tolerance, or cardiac function in patients with COPD. These important parameters have been well studied in congestive cardiac failure. ${ }^{2}$ We look forward to seeing the results of similar studies in oedematous respiratory failure.

A G STEWART on behalf of authors
Clinic $2 A$,

Addenbrookes Hospital Cambridge CB2 4fF,

1 Farber MO, Weinberger MH, Roberson GL, Fineberg NS. The effects of angiotensin converting enzyme inhibition on sodium handling in patients with advanced chronic obstructive pulmonary disease. Am Rev Respir Dis 1987, 136:862-6.

2 Bounhoure JP, Bottineau G, Lechat P, Garnham $J$, Lapeyre G. Value of perindopril in the treatment of chronic congestive cardiac failure Clin Exp Theory Practice 1989;A11, S1:575-86.

\section{BOOK NOTICES}

Therapeutics in Respiratory Disease. 2nd edition. P J Barnes, K F Chung, T W Evans, S G Spiro (Pp 174; $£ 40.00)$. Edinburgh: Churchill Livingstone, 1994. 443041342

This is a new book covering the management and treatment of lung disease. The four authors are from the National Heart and Lung Institute, with contributions from $D$ Rob Miller and Dr Norman Horne on AIDS and tuberculosis, respectively. The chapter headings cover obstructive airways diseases and respiratory failure, respiratory infections including opportunistic infections, AIDS and tuberculosis, lung cancer, pulmonary vascular disease, and drug-induced pulmonary disease, together with an initial chapter on general principles of drug treatment and a further chapter on symptom management. The chapters are focused on individual drugs and on the diseases and their management. The book has a pleasing format with several boxes in each chapter to highlight important points.

The style and value of the book varies considerably between chapters. Those on opportunistic infection, tuberculosis, and cancer are clear, practical, and authoritative, giving details of the drugs available, followed by management strategies, and what to do when complications occur - for example, hepatitis with antituberculous drugs or failure to respond to treatment for Pneumocystis carini pneumonia. These are chapters that I will use (and have already used) for practical help when problems arise. They pull together areas which the non-specialist finds difficult to keep track of such as the role of chemotherapy in non-small cell lung cancer. The reader is given a clear picture of the state of the art.

Some of the other chapters were less satisfactory since there seemed to be some confusion between what might be expected theoretically and what had been demonstrated in clinical practice. These chapters were less well organised and the writing sometimes had a slightly "off the cuff" feel to it, with repetition and, occasionally, contradictions. Some statements could be questioned - for example, administration of heparin for five days for a deep vein thrombosis, or the statement that patients with pure respiratory failure do not develop bicarbonate retention (surely this is a function of time of increased carbon dioxide levels). I was also concerned by an action plan for asthma that allows PEF to fall to $30 \%$ of normal before suggesting that medical attention should be sought.

There is a tendency to adopt a technique used by medical students which is to say that a certain treatment can be used - what the reader wants to know is how, when, and where it should be used and whether there is evidence that it is beneficial. Many conditions are not covered such as lung abscess, empyema, sarcoidosis, fibrosing alveolitis, allergic alveolitis, and many less common conditions. There is relatively little on bronchiectasis and, more surprisingly perhaps, no discussion of the role of nutrition or inhaled amiloride, steroids, or DNase in the management of cystic fibrosis.
There is a need for a book on therapeutics in respiratory disease and this book succeeds for some diseases. If the book as a whole is to fulfil this niche the style needs to be more uniform and the reader needs to be confident that most diseases and most aspects of treatment will be covered. There are many reviews on research in progress and, although an outline of drugs in the early stages of de- $x$ velopment is valuable, this must be distinguished from treatment that is of proven value. - AET

Respiratory Disease in Children: Diagnosis and Management $G M$ Loughlin and H Eigen. (Pp 870; $£ 112.00)$. Baltimore: Williams and Wilkins, 1994. 0683051903.

This is a sizeable but interesting book of 870 pages which is a little different from other 0 textbooks on the topic of respiratory disease in children. Sections include scientific foundations, diagnostic techniques, clinical assessment, and common and unusual o respiratory disorders, with an innovation for 을 books of this type in that a relatively small section is devoted to common problems such $D$ as noisy breathing, cough, haemoptysis, and chest pain. The majority of the contributors are from North America and it appears to have developed out of a paediatric pulmonology of training programme; the foreword heralds the book as a product of second generation paediatric lung specialists. The style of individual chapters varies widely between standard reviews - for example, on airway mechanics and respiratory control - to chapters which $\Omega$ are more like review articles in medical journ- $\overrightarrow{\vec{\beta}}$ als - such as the chapter on the development of airways responsiveness and its relationship to asthma. There are some ambiguities between contributors - for example, in the origins of lung disease the genetic versus the acquired hypothesis for chronic obstructive lung disease in adult life is reviewed, whereas ? in the next chapter on prevention of lung disease the contributors plump for early respiratory infection as the most likely early $\delta$ life cause of adult chronic obstructive lung disease.

Contributions on diagnostic tests are not very different from other well established texts. There is no mention of infant lung function testing which one would expect in a $N$ book of this size. The imaging chapter is $N$ particularly helpful with many chest radio- N graphs supported by secondary imaging $\omega$ investigations. A very useful section on the principles of treatment includes the statistical 0 background to clinical trials and issues such $\underset{\Phi}{\overparen{D}}$ as identification of correct sample size. The $\stackrel{\mathscr{S}}{?}$ list of medical treatments and drugs used is North American in flavour and will require $\bar{O}$ translation for the European and Australasian $\mathbb{D}$ markets. There are useful sections on home care, nutritional management, and chest $ळ$ physiotherapy. Overall this is an interesting addition to the growing number of paediatric 8 pulmonology textbooks. I would certainly recommend it for those with a special interest in paediatric pulmonology and, bearing in mind the origins of the work, this is probably? what the authors intended. With a price of $£ 112$ it is likely that this book will only find its way into the hands of those individuals who are planning to make a career in paediatric pulmonology or those responsible for training programmes. - PJH 